\title{
A child with resistant Kawasaki disease successfully treated with anakinra: a case report
}

\author{
J. Sánchez-Manubens ${ }^{1,2^{*}}$, A. Gelman ${ }^{3}$, N. Franch ${ }^{3}$, S. Teodoro ${ }^{4}$, J. R. Palacios ${ }^{4}$, N. Rudi ${ }^{5}$, J. Rivera ${ }^{3}$ and J. Antón ${ }^{2}$
}

\begin{abstract}
Background: Kawasaki disease (KD) is an acute self-limited systemic vasculitis of unknown etiology. Intravenous immunoglobulin (IVIG) is an effective treatment and decreases the risk of cardiac complications to less than 5\%. In spite of its effectiveness, some children do not respond to this therapy and still develop coronary aneurysms (CAA). The optimal treatment for IVIG non-responsive patients remains controversial although corticoids have been suggested to be an effective treatment in some patients. For those patients still resistant to IVIG and corticoids, interleukin-1 receptor antagonists (IL-1RA) such anakinra could be an alternative.

Case presentation: We present a 3 year-old Caucasian patient with KD without cardiac complications but with important resistance to treatment. After becoming resistant to IVIG and corticoids, anakinra proved to be an effective treatment.

Conclusions: To our knowledge, this is the first report of the utility of IL-1RA in refractory KD without coronary impairment. The patient fulfilled the classical criteria for KD and, after becoming resistant to first and second line treatments, anakinra proved to be an effective treatment. Further studies are required to determine if this is an effective treatment option for other cases of resistant Kawasaki disease.
\end{abstract}

Keywords: Kawasaki disease, IVIG resistance, Anakinra, IL1 blockade, Case report

\section{Background}

$\mathrm{KD}$ is an acute self-limited systemic vasculitis of unknown etiology presenting predominantly in toddlers and children under 5 years old. Diagnosis is based on clinical criteria including fever, exanthema, conjunctivitis, changes in hands and feet, erythema of oral mucosa and lips and cervical lymphadenopathy. Prognosis depends on the extent of cardiac involvement; CAA develop in $20-25 \%$ of untreated patients and these may lead to myocardial infarction and sudden death if proper treatment with IVIG is not administered within the first 10 days of illness $[1,2]$. Early treatment with IVIG decreases the risk of cardiac complications to less than $5 \%$. In spite of its effectiveness, some children do not

* Correspondence: jsanchez@tauli.cat

${ }^{1}$ Pediatric Rheumatology Unit, Pediatrics Department, Hospital Parc Taulí

Sabadell, Barcelona, Spain

${ }^{2}$ Pediatric Rheumatology Unit, Pediatrics Department, Hospital Sant Joan de

Déu Esplugues, Barcelona, Spain

Full list of author information is available at the end of the article respond to this therapy and still develop CAA. Although the optimal treatment for IVIG non-responsive patients remains controversial, adding steroids to the 2nd IVIG dose has proven to be effective to reduce the incidence of CAA and improve the prognosis of resistant $\mathrm{KD}$ when administered in patients fulfilling IVIG resistance criteria in the Kobayashi scoring system [3, 4]. For those who present a lack of response to this 2nd step of treatment, other approaches have been described, such as infliximab [5], plasma exchange [6] or cyclosporine [7]. In the last years some reports have suggested the role of IL-1RA in the treatment of severe or resistant cases of KD $[8,9]$. We present a case of a IVIG and steroids resistant KD successfully treated with IL-1RA (anakinra).

\section{Case presentation}

We present a previously healthy 3 year-old Caucasian girl, who was admitted with persistent fever for 5 days, generalized rash, non-purulent conjunctivitis, labial and lingual erythema and swollen feet. On admission, the girl 
had significant irritability. Blood tests showed normal hemoglobin, white blood cells and platelets, high Creactive protein (CRP - $14 \mathrm{mg} / \mathrm{dL}$ ) and high transaminases (AST $168 \mathrm{U} / \mathrm{L}$, ALT $86 \mathrm{U} / \mathrm{L}$ ). Fulfilling the KD classical criteria, KD was diagnosed and treatment with IVIG $(2 \mathrm{~g} / \mathrm{kg})$ and aspirin $(100 \mathrm{mg} / \mathrm{kg})$ was initiated. Echocardiography two days after admission showed mild mitral and tricuspideal regurgitation but no CAA. Electrocardiogram was normal.

Despite the initial IVIG treatment, fever, rash, conjunctivitis and labial and lingual erythema remained, and 2 additional IVIG doses and 2 metilprednisolone $(30 \mathrm{mg} / \mathrm{kg})$ pulses were administered in the subsequent days. Eight days after admission, fever disappeared and analytical features normalized.

Maintenance treatment with oral prednisone $(0.5 \mathrm{mg} /$ $\mathrm{kg} /$ day) was initiated but, on day 11 after admission, fever recurred with important irritability, exanthema and hand and feet desquamation. An important increase on CRP $(16.5 \mathrm{mg} / \mathrm{dL})$, ESR $(126 \mathrm{~mm} / \mathrm{h})$ and platelets $(808000)$ was registered and there was a decrease in hemoglobin (7.5 g/dL) (Fig. 1). Echocardiography did not show changes. Another 2 metilprednisolone pulses were administered without response. On day 14 after admission (19 days since onset), due to conventional treatment failure and having ruled out, together with the patient's parents, the use of other intravenous treatments such as infliximab, IL-1RA was initiated (anakinra $-2 \mathrm{mg} / \mathrm{kg}$ subcutaneous once a day for 14 days). Fever and irritability disappeared within hours and CRP, ESR, platelets and hemoglobin became normal in the subsequent blood tests ( $24 \mathrm{~h}$ and 5 days after treatment initiation).

Six days after anakinra was initiated the patient was discharged. Treatment was maintained for 2 weeks combined with aspirin. No relapses appeared after anakinra discontinuation and subsequent blood tests and echocardiographies at weeks 2,8 and 16 after discharge were normal (no changes on coronary arteries sizes have been recorded over time and z-scores have maintained between
1 and $1.5 \mathrm{SDs}$ ). The patient did not experience any side effects or complications during or after the use of anakinra.

\section{Discussion}

In the last years, some studies have suggested an important role for interleukin 1 (IL-1) in the pathogenesis of KD [10]. Genome-Wide Association Studies (GWAS) have also found functional SNPs in ITPKC and CASP3 genes that are associated with an increased risk of unresponsiveness to IVIG therapy [11]. Lee et al. suggested, using a mouse model of KD, that IL-1 receptor-deficient mice were protected from induced coronary lesions. Furthermore, daily injections of the IL-1RA prevented induced coronary lesions in normal mice [12].

Another study developed in a cohort of Taiwanese children with KD, found a significant increase in IVIG resistance risk in those patients with the IL-1B -511 TT and IL-1B $-31 \mathrm{CC}$ genotypes and the diplotype TC/TC in the IL-1 family of genes [13]. These results suggest an important genetic association between IL-1 and failure of initial IVIG therapy and support the previous findings that IL-1 secretion is associated with IVIG treatment in $\mathrm{KD}[14,15]$.

Anakinra is a recombinant antagonist of the IL-1 receptor used successfully to treat systemic onset juvenile idiopathic arthritis. Regarding the clinical application of the exposed findings on the role of IL-1 in the pathogenesis of KD, two clinical trials are being held in Europe and the USA and two case reports on KD patients treated with anti-Il-1 have been reported $[8,9]$. Both papers describe severe cases of KD, one of them complicated with a macrophage activation syndrome (MAS), that were resistant to multiple IVIG and prednisolone pulses and improved drastically after the administration of anakinra with analytical normalization and complete reversal of the echocardiogram changes. We present a case report in which the patient fulfilled the classical criteria for KD but had no cardiac or severe complications and, after becoming resistant to first and second line

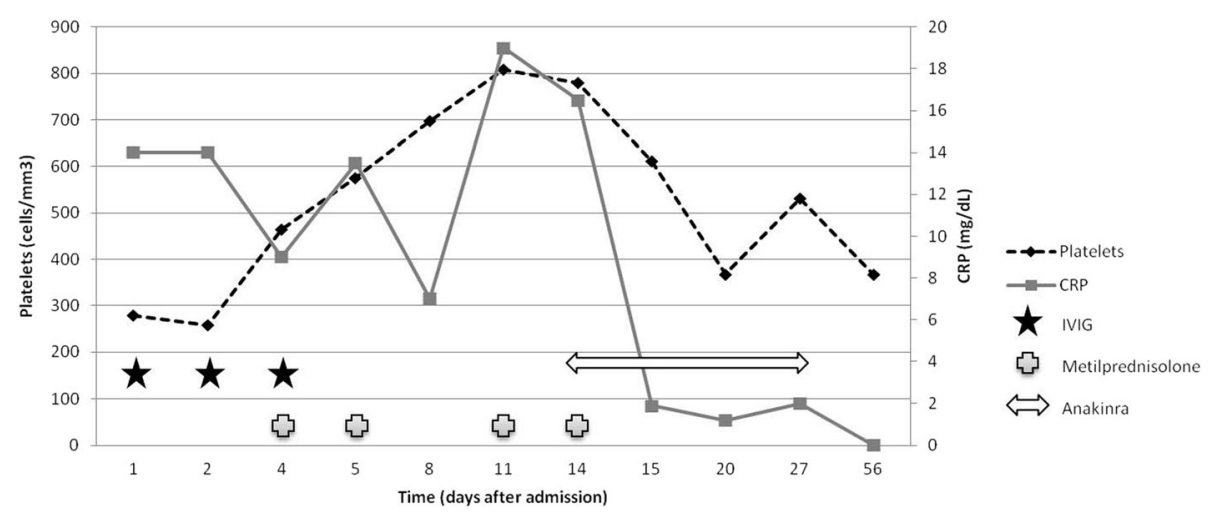

Fig. 1 Time course of $C$ reactive protein (CRP), platelets and treatment 
treatments, IL-1R blockade proved to be an effective treatment. Further studies are required and are being held to determine if this is an effective treatment option for all cases of resistant Kawasaki disease.

\section{Conclusion}

To our knowledge, this is the first report on the utility of IL-1RA in refractory KD without coronary impairment or MAS. The patient fulfilled the classical criteria for KD and, after becoming resistant to first and second line treatments, IL-1R blockade proved to be an effective treatment without side effects or complications. Further studies are required to determine if this is an effective treatment option for other cases of resistant Kawasaki disease.

\section{Abbreviations}

ALT: Alanine aminotransferase; AST: Aspartate aminotransferase; CAA: Coronary aneurysms; CRP: C-reactive protein; ESR: Erythrocyte sedimentation rate; IL1RA: Interleukin-1 receptor antagonists; IVIG: Intravenous immunoglobulin; KD: Kawasaki disease; MAS: Macrophage activation syndrome

\section{Acknowledgments}

We would like to thank all the staff in the Pediatrics Department of Hospital Parc Tauli and Rheumatology Unit of Hospital Sant Joan de Déu for their help in the resolution of the case.

\section{Funding}

No external funding has been obtained for the realization or publication of the article.

\section{Availability of data and materials}

The dataset supporting the conclusions of this article is included within the article.

\section{Authors' contributions}

All authors have read and approved the manuscript. They have contributed to the article as follows: JSM: carried out the data collection, drafted the initial manuscript, obtained the patient consent form and approved the final manuscript as submitted. AG, NF, ST, JRP and NR: helped with the resolution of the clinical case and reviewed and revised the manuscript and approved the final manuscript as submitted. JR and JA: reviewed and revised the manuscript and approved the final manuscript as submitted.

\section{Competing interests}

The authors declare that they have no competing interests. The authors of the article state that they don't have conflict of interest to disclose. They are not shareholding in a company, they have not received any grant or consultancy fee from a company whose product features in the submitted manuscript or manufactures a competing product.

\section{Consent for publication}

Written informed consent was obtained from the parents for publication of this case report.

\section{Ethics approval and consent to participate}

Ethics approval and consent was no needed for the use of anakinra in the patient. It was used as an off-label medication with the parents and the Pharmacy Department consent. Written informed consent was obtained from the parents for publication of this case report.

\section{Publisher's Note}

Springer Nature remains neutral with regard to jurisdictional claims in published maps and institutional affiliations.

\section{Author details}

${ }^{1}$ Pediatric Rheumatology Unit, Pediatrics Department, Hospital Parc Taulí Sabadell, Barcelona, Spain. ${ }^{2}$ Pediatric Rheumatology Unit, Pediatrics Department, Hospital Sant Joan de Déu Espluques, Barcelona, Spain. ${ }^{3}$ Pediatrics Department, Hospital Parc Taulí Sabadell, Barcelona, Spain. ${ }^{4}$ Pediatric Cardiology Unit, Pediatrics Department, Hospital Parc Taulí Sabadell, Barcelona, Spain. ${ }^{5}$ Pharmacy Department, Hospital Parc Taulí Sabadell, Barcelona, Spain

Received: 28 April 2016 Accepted: 29 March 2017

Published online: 08 April 2017

\section{References}

1. Newburger JW, Takahashi M, Gerber MA, et al. Diagnosis, treatment, and long-term management of Kawasaki disease: a statement for health professionals from the committee on rheumatic fever, Endocarditis, and Kawasaki disease, council on cardiovascular disease in the young, American Heart Association. Pediatrics. 2004;114:1708-33. doi:10.1542/peds.2004-2182.

2. Sánchez-Manubens J, Bou R, Anton J. Diagnosis and classification of Kawasaki disease. J Autoimmun. 2014;48-49:113-7. doi:10.1016/j.jaut.2014.01.010.

3. Kobayashi T, Kobayashi T, Morikawa A, et al. Efficacy of intravenous immunoglobulin combined with prednisolone following resistance to initial intravenous immunoglobulin treatment of acute Kawasaki disease. J Pediatr. 2013;163:521-6. doi:10.1016/j.jpeds.2013.01.022.

4. Brogan P, Levin M. Intravenous immunoglobulin plus corticosteroids prevent coronary artery abnormalities in Kawasaki disease. Evid Based Med. 2013;18:217-8. doi:10.1136/eb-2013-101264.

5. Son MB, Gauvreau K, Burns JC, et al. Infliximab for intravenous immunoglobulin resistance in Kawasaki disease: a retrospective study. J Pediatr 2011;158:644-649. e1. doi:10.1016/j.jpeds.2010.10.012

6. Sonoda K, Mori M, Hokosaki T, Yokota S. Infliximab plus plasma exchange rescue therapy in Kawasaki disease. J Pediatr 2014;164:1128-1132. e1. doi:10.1016/j.jpeds.2014.01.020.

7. Tremoulet AH, Pancoast P, Franco A, et al. Calcineurin inhibitor treatment of intravenous immunoglobulin-resistant Kawasaki disease. J Pediatr 2012;161: 506-512. e1. doi:10.1016/j.jpeds.2012.02.048.

8. Cohen S, Tacke CE, Straver B, et al. A child with severe relapsing Kawasaki disease rescued by IL-1 receptor blockade and extracorporeal membrane oxygenation. Ann Rheum Dis. 2012;71:2059-61. doi:10.1136/annrheumdis2012-201658.

9. Shafferman A, Birmingham JD, Cron RQ. High dose Anakinra for treatment of severe neonatal Kawasaki disease: a case report. Pediatr Rheumatol Online J. 2014;12:26. doi:10.1186/1546-0096-12-26.

10. Hoang LT, Shimizu C, Ling L, et al. Global gene expression profiling identifies new therapeutic targets in acute Kawasaki disease. Genome Med. 2014:6:541. doi:10.1186/s13073-014-0102-6.

11. Onouchi $Y$, Suzuki $Y$, Suzuki H, et al. ITPKC and CASP3 polymorphisms and risks for IVIG unresponsiveness and coronary artery lesion formation in Kawasaki disease. Pharmacogenomics J. 2013;13:52-9. doi:10.1038/tpj.2011.45.

12. Lee $Y$, Schulte DJ, Shimada $K$, et al. Interleukin-1 $\beta$ is crucial for the induction of coronary artery inflammation in a mouse model of Kawasaki disease. Circulation. 2012:125:1542-50. doi:10.1161/CIRCULATIONAHA.111.072769.

13. Weng K-P, Hsieh K-S, Ho T-Y, et al. IL-1B polymorphism in association with initial intravenous immunoglobulin treatment failure in Taiwanese children with Kawasaki disease. Circ J. 2010;74:544-51. http://www.ncbi.nlm.nih.gov/ pubmed/20081319. Accessed 27 Jan 2016.

14. Leung DY, Cotran RS, Kurt-Jones E, et al. Endothelial cell activation and high interleukin-1 secretion in the pathogenesis of acute Kawasaki disease. Lancet (London, England). 1989;2:1298-302. http://www.ncbi.nlm.nih.gov/ pubmed/2480498. Accessed 27 Jan 2016.

15. Suzuki $H$, Uemura $\mathrm{S}$, Tone $\mathrm{S}$, et al. Effects of immunoglobulin and gammainterferon on the production of tumour necrosis factor-alpha and interleukin-1 beta by peripheral blood monocytes in the acute phase of Kawasaki disease. Eur J Pediatr. 1996;155:291-6. http://www.ncbi.nlm.nih. gov/pubmed/8777922. Accessed 27 Jan 2016. 